\title{
Who is at risk? Periodontal disease risk analysis made accessible for the general dental practitioner
}
IN BRIEF
- Fully explore socio-demographic factors of patients and their relevance to periodontitis.
- Use an easy-to-apply scoring system to grade a patient's periodontal risk.
- Tailor your preventive programme to effectively eliminate possible risk factors.
- Learn about preventing/minimising systemic diseases by treating and preventing periodontitis.

\author{
A. J. Cronin, ${ }^{1}$ N. Claffey ${ }^{2}$ and L. F. Stassen ${ }^{3}$
}

VERIFIABLE CPD PAPER

\begin{abstract}
The use of odds ratio $(\mathrm{OR})$ in risk analysis is considered a useful means of cross-comparing risk factors by which a disease is influenced. This article outlines the interpretation of reported ORs with respect to periodontal disease, highlighting those factors that are most deserving of consideration. The claim that periodontal disease is implicated in some serious systemic diseases is investigated with respect to the evidence. Data retrieval was carried out, focusing primarily on ORs for factors shown to be positively correlated with the incidence of periodontal disease. Using the available data, a risk evaluation scoring system was proposed (the Cronin/Stassen BEDS CHASM scale). The results of the evaluation support the view that hygiene therapy, smoking cessation and control of the glycaemic state offer the largest and most easily achieved reduction in risk with respect to attachment loss. The literature on systemic diseases indicates that the mechanisms with which periodontitis may interact seem biologically plausible, compellingly so in the cases of coronary heart disease and diabetes mellitus. However, statistical evidence to confirm these suggested interactions is equivocal, with the data retrieved in some instances being less than decisive. Further investigation is recommended. Glossary: AL: attachment loss, BL: bone loss, BMI: body mass index, $\mathrm{Cl}$ : confidence interval, CHD: coronary heart disease, CVD: cardiovascular disease, IE: infective endocarditis, OR: odds ratio, SES: socioeconomic status, HAART: highly active anti-retroviral therapy.
\end{abstract}

\section{INTRODUCTION}

Periodontitis is a complex disease entity with a layered and multifactorial aetiology. ${ }^{1-3}$ The causative agents implicated in the disease are often not enough to cause the common clinical scenario of inflammation, bleeding and ultimately, attachment loss (AL).,5 This observation has led researchers to search for risk factors that differentiate those affected from those spared.

Risk is the 'possibility of injury', or the likelihood of developing a condition or health change over a time period. ${ }^{6}$ An

\footnotetext{
${ }^{1 *}$ Periodontology, ${ }^{2}$ Dean, Professor of Periodontology, ${ }^{3}$ Professor of Oral and Maxillofacial Surgery, Dublin Dental School and Hospital, Lincoln Place, Dublin 2, Ireland/St. James's Hospital, James's St. Dublin 8, Ireland ${ }^{*}$ Correspondence to: Aaron Cronin, 10 Cairnfort, Enniskerry Road, Stepaside, Co. Dublin, Ireland Email: cronin.aaron@gmail.com
}

\section{Refereed Paper}

Accepted 24 April 2008

DOI: $10.1038 /$ sj.bdj.2008.653

${ }^{\bullet}$ British Dental Journal 2008; 205: 131-137 odds ratio (OR) is a "comparison of the presence of a risk factor for disease in a sample of diseased subjects and nondiseased controls'. The term 'risk factor' implies that for any disease, there are influences associated with an increased/ decreased likelihood of exposure. ${ }^{8}$

In order to test a putative risk factor for its association with periodontitis, the following four-step risk assessment model is recommended: ${ }^{9,10}$

1. Identify potential factors with a biologically plausible aetiology that exhibit a statistically significant OR in cross-sectional analysis ${ }^{11,12}$

2. Demonstrate through multivariate testing that the putative risk factor retains this significance when confounded by other known influences

3. The model should be assessed and corroborated by other studies, with the results reflecting the expectations

4. Targeted treatment addressing the factor in question should lead to a reduction in incidence of the associated disease.
The first hurdle in establishing an association between an exposure and a disease is to express the relationship between the two in numerical form, with a statistic that can be interpreted. Odds ratio is an example of such an expression (Fig. 1)..$^{13}$

Following an investigation, those factors with a significant association with periodontitis, despite the presence of confounding factors, require a targeted treatment phase to allow their status as true risk factors to be confirmed. Only three such factors have been qualified in this manner: specific plaque bacteria, smoking and poorly controlled diabetes. ${ }^{10}$ This paper reviews the evidence regarding putative periodontal risk factors and how it should dictate the treatment of this pathological process. Odds ratios from published clinical trials are included as evidence, having been selected from a PubMed search engine with preference having been given to articles of a multifactorial nature. All claims were validated through analysis 
of the odds ratios and their corresponding confidence intervals.

\section{RISK FACTORS IMPLICATED IN PERIODONTAL DISEASE}

\section{Socio-demographic factors}

\section{Age}

Age lacks any proven physiological impact that links it to periodontitis. In 1993, Pfeilschifter suggested that older individuals suffer bone loss (BL) as a result of down-regulation of osteoblasts and mitogens in repair. ${ }^{14}$ Strictly speaking, periodontal disease is ubiquitous amongst older age groups. Periodontitis progresses discontinuously throughout life and the prevalence in cross-sectional studies in advanced age has more to do with a lifetime of AL than any increased incidence in the elderly. ${ }^{5,15,16}$ Nonetheless, in cross section, it is clear that older age groups (65+ years) have increased AL, multivariate studies having demonstrated ORs for AL as high as 9.01 (95\% CI: 5.8613.89), constituting the most significant risk indicator in periodontal disease. ${ }^{17}$ It is recognised as a product of disease experience throughout life rather than something intrinsic with being older. ${ }^{18}$ With all studies unanimous that with increased age comes increased disease, older age groups will exhibit more periodontal destruction practically every time.

\section{Gender}

In a case-control environment, gender seemingly exhibits a predictable influence on the prevalence of periodontitis. This influence, however, is confounded by factors such as smoking, supporting the view that this predisposition of males is primarily behavioural in nature. ${ }^{19}$ Two possibilities have been suggested by Grossi in 1995 as to why males are more predisposed to AL (OR 1.36, 95\% CI: 1.06-1.76). Being male may have some physiological impacts on periodontal health, or may exert influence on oral health via a stereotypically blasé attitude towards oral hygiene, with a decreased likelihood to seek dental care. ${ }^{17}$ Horning's study in 1992 found similar results, reporting males to have increased risk of moderate or advanced periodontitis (OR 1.78, 95\% CI: 1.42-2.46). ${ }^{20}$ Male gender deserves consideration in a risk model

\begin{tabular}{|c|c|c|}
\hline & Exposed & Non-Exposed \\
\hline Group A - Diseased & $x$ & $a$ \\
\hline Group B - Control & $y$ & $b$ \\
\hline
\end{tabular}

Two groups, A and B, are compared in a cross-sectional survey for an exposure, odds are calculated for the exposure in each group.

Odds of Exposure in Group A - x/a

Odds of Exposure in Group B - $y / b$

In order to compare the outcomes in each group, these two odds are expressed as a ratio:

$\underline{(x / a)}$ or simply $\quad \underline{x b}$

$(y / b) \quad y a$

This odds ratio $(\mathrm{OR})$ can range from 0 to Infinity.

An $O R>1$ implies that an exposure is more likely in Group A. The disease status of those in Group A can be said to be positively correlated with the exposure being investigated.

An $\mathrm{OR}<1$ implies that exposure is less likely in Group A. The disease status of those in Group A can be said to be negatively correlated with the exposure under investigation.

Where an $\mathrm{OR} \approx 1$, it implies that exposure is equally likely in both Groups $\mathrm{A}$ and $\mathrm{B}$. The disease status under investigation cannot be correlated with the exposure in question.

Fig. 1 Brief explanation of the calculation and interpretation of odds ratio (OR) ${ }^{1}$

for periodontal disease, the effects of which must be addressed through behaviour modification.

\section{Socioeconomic Status \\ (SES)/Education}

Indices for assessing SES have been used, ranging from income level to education level. ${ }^{8,17}$ All factors show a positive correlation, with periodontal disease prevalence attributed to a 'differential access to resources and opportunities that may influence preventive behaviours', with OR as high as 3.10 (95\% CI: 1.64-6.03), indicating a statistically significant divide between those that have attended some 3rd level education and those who have not. ${ }^{15,21}$ Other multivariate clinical analyses have put the OR even higher, though it is perhaps difficult to completely separate SES from other, more physiological variables. ${ }^{22}$ Socioeconomic status may represent a measure of personal drive and motivation, and may impact on the quality of oral hygiene habits, and as such represents a valid risk indicator.

\section{Ethnicity}

Studies have observed a correlation between race and periodontitis. Odds ratios with Caucasians have placed others (Pacific Islanders, Native Americans, Asians) at an increased risk of AL (OR 1.72, 95\% CI: 1.26-2.35) and bone loss (BL, OR 2.4, 95\% CI: $1.21-1.79) .{ }^{17,20}$ Periodontitis appears to affect other ethnicities even if living in Europe or USA and even across socioeconomic divisions, though some amount of compounding is likely to be present in the implicated factors. ${ }^{21}$ Despite the excuses given, a risk of increased periodontitis exists for these ethnicities.

\section{Hereditary or acquired conditions}

\section{Diabetes}

The reduced function of the polymorphonuclear leukocyte in diabetes is thought to contribute to the increased prevalence of periodontal disease. This dysfunction in chemotaxis, adherence and phagocytosis is thought to be fundamental in the host's predisposition to periodontal infection..$^{23}$ In addition, the formation of advanced glycosylation end-products (AGEs, Fig. 2) is thought to be the source of many diabetic complications via impaired collagen production and metabolism. Non-enzymatic glycosylation of numerous body proteins 
(collagen included) in the hyperglycaemic environment leads to an induced hyperreactive state with oversecretion of inflammatory mediators such as interleukin-1 (IL-1) and tumour necrosis-factor (TNF)- $\alpha$, rendering the patient more susceptible to tissue destruction. ${ }^{24}$

Long recognised as a risk factor, the evidence has shown that uncontrolled diabetes strongly correlates with increased BL. ${ }^{25-27}$ Studies have also shown that infections observed in periodontitis can lead to insulin resistance and poor diabetic control. ${ }^{28,29}$

Interpreting the significance of diabetes in multivariate models is difficult because very high numbers of individuals in a sample population would be needed to allow interpretation on a broader perspective. ${ }^{16}$ When controlled for confounding factors in one such study, however, diabetes was correlated with AL at an OR of 2.32 (95\% CI: 1.17-4.60), making it the only systemic disease with a positive correlation..$^{30}$ The implication is that when diabetes is poorly controlled, the improvement is less marked for initially deep pockets. Where control of the condition is established, the clinical outcome is similar to that of non-diabetics. ${ }^{31}$ The evidence thus suggests that controlling this condition can be the decisive factor in the effectiveness of surgical and non-surgical treatment.

\section{Cardiovascular disease (CVD)}

Studies have examined the relationship between CVD and periodontitis. Suggestions that angina pectoris predisposes a person to periodontal disease are equivocal. Angina appeared to exhibit some influence in isolation. However, when controlled with other factors such as smoking and age, the effect was deemed insignificant. ${ }^{30}$ The converse is also proposed, periodontitis having been thought to influence CVD. It is proposed that a common hyperinflammatory monocyte phenotype may be implicated in both, making periodontitis an indicator, rather than cause, of CVD. ${ }^{32}$

\section{Obesity}

Studies have looked at the interaction between obesity and periodontal disease. Al-Zahrani in 2003 demonstrated that a BMI $>30 \mathrm{~kg} / \mathrm{m}^{2}$ predisposes a person to more severe AL (OR of 1.76, 95\% CI: 1.19-2.61), figures which other authors have found consistent. ${ }^{33,34}$ Young people especially were implicated - having a high waist circumference carried with it an OR of 2.27 (95\% CI: 1.480 to 3.487). ${ }^{33}$ Although the physiology remains unclear, the relationship between the conditions is strong, and deserves consideration as part of the risk model.

\section{HIV/immunesuppression}

Recent literature has questioned the link between HIV and progression of periodontitis, which hitherto has been accepted. $^{35}$ Some have claimed that highly-active anti-retroviral therapy has limited periodontal disease progression in HIV-positive patients. ${ }^{36}$ Studies revealing no difference in periodontal status between HIV-seropositive and HIV-seronegative refute this, concluding that the "extent and severity of periodontal disease among HIV-infected individuals may be less than hitherto thought. ${ }^{37}$ Treatment with HAART has indicated that seropositivity with HIV doesn't in itself constitute a serious risk of periodontal disease.

\section{Osteoporosis/osteopaenia}

Individual reports have conflicted regarding the association between osteoporosis and periodontitis. Both conditions involve BL and may share common aetiological pathways. Studies have reported significant association between the diseases, whereas others have failed to find any interaction. ${ }^{38,39}$ Studied almost exclusively in elderly women, the association has proven weak.

\section{Behavioural factors}

\section{Smoking}

The deleterious effects of smoking on periodontal health have been well-documented since 1947, when increased prevalence of acute necrotising ulcerative gingivitis was noted. ${ }^{40}$ The synergy of toxic effects on immune cell chemotaxis, disruption of the aerobic flora, inhibition of osteoblasts and vasoconstriction of gingival blood vessels favours severe AL and alveolar BL. ${ }^{30,41}$ With ORs for AL in the multivariate model for light smokers comparable to those for uncontrolled diabetes (OR 2.05, 95\% CI: 1.47-2.87), the correlation is ubiquitous in the literature. ${ }^{30}$ Increased smoking pack years demonstrate a dose-response effect, resulting in OR for heavy smokers of 4.75 for AL (95\% CI: 3.28-6.91). ${ }^{30}$ Less bleeding on probing is observed, misleading the clinical picture with regard to deep pockets and bony defects. ${ }^{42}$ Smoking is also a cause of impaired healing after periodontal treatment. Impaired pocket

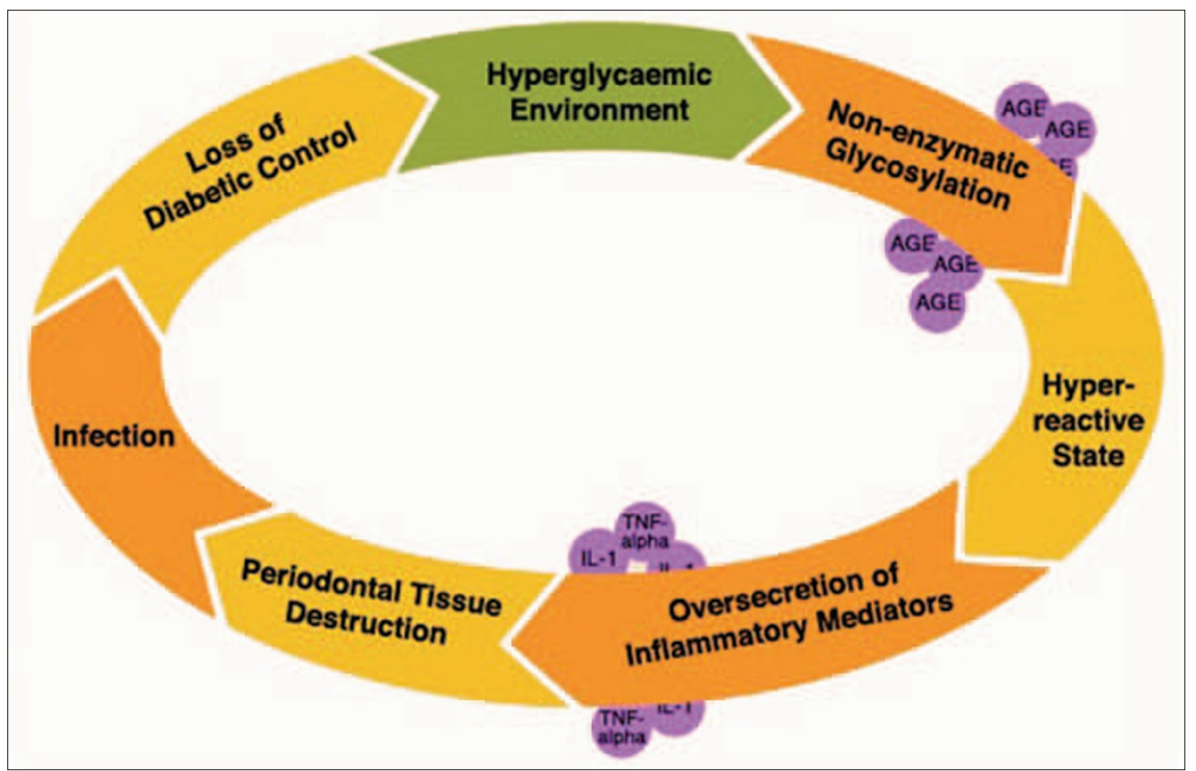

Fig. 2 Cycle of periodontal destruction in diabetes ${ }^{24}$

TNF-alpha = Tumour Necrosis Factor- alpha, IL-1 = Interleukin-1, AGE = Advanced Glycosylation End Products Summary - A hyperglycaemic environment will lead to uncontrolled glycosylation of blood proteins, including collagen and haemoglobin. Hyper-reactive Glycated proteins (AGEs) of the immune system are prone to oversecrete inflammatory mediators in response to periodonta pathogens, putting the host at an increased risk of oxidant-stress-related periodontal attachment loss. With progressive attachment loss comes a greater propensity for infection of the periodontium, which can lead to insulin resistance through dysregulation of diabetic control hormones. 
reduction in non-surgical treatment stems from reduced recession of the gingiva in the absence of oedema. This difference averages $0.5 \mathrm{~mm}$ between smokers and non-smokers. Post-surgery, flap revascularisation is affected by nicotine and smoke. ${ }^{43}$ With every study stacking their evidence firmly in one camp, the establishment of smoking as one of the few true risk factors is without contention.

\section{Periodontal risk factors}

\section{Specific bacteria in subgingival plaque}

Three bacteria have been implicated (according to Koch's postulates) as causative agents for periodontitis. The presence of Tannerella. forsythensis (OR 2.45, 95\% CI: 1.87-3.24) and Porphyromoras. gingivalis (minimum OR 1.59, 95\% CI: 1.11-2.25) are both influential. ${ }^{30,44}$ Aggregatibacter. actinomycetemcomitans is also implicated. ${ }^{21}$ The bacteria are present before the disease onset, and increased numbers are correlated with its progression, if untreated. ${ }^{45}$ Possibly more significant than the actual colonisation is that a high load of these organisms increases the likelihood of deep pocketing. ${ }^{46}$ Reduction of load in affected sites leads to attachment gain, whereas progression was noted in sites with residual colonies. ${ }^{47}$ Such studies have formed the basis of modern treatments aimed at plaque control. If proper hygiene is instituted and bacterial load kept low, disease progression can be minimised. Many putative risk factors have been explored and expressed as a function of oral hygiene and plaque control, underscoring the importance of these agents.

\section{Crowding}

It has been theorised that malocclusion and crowding offers an environment that favours plaque accumulation, representing a periodontal disease risk. Several studies, using various indices to quantify this parameter, have failed to report any such association. ${ }^{48,49}$

\section{Psychological and cognitive factors}

\section{Stress}

Stress is thought to manifest in the periodontium through behavioural changes, such as increased smoking and poorer oral hygiene..$^{50}$ Subjects demonstrating poor coping behaviour under financial strain exhibited more AL (OR 2.24, 95\% CI: 1.15-4.38) than those better able to cope, even after these were controlled. ${ }^{51}$ Job stress and health stress were also found to be significant variables. ${ }^{52}$ Stress levels warrant inclusion in the risk model, though the cause and effect relationship needs to be clarified.

\section{Bruxism}

Evidence that dynamic occlusal interferences influence AL has been reported, though the influence is regarded as weak. ${ }^{53}$ Other studies have demonstrated the mutually exclusive clinical pictures of the two conditions. ${ }^{54}$ The relationship between the two conditions should therefore be considered weak.

\section{Host defence factors}

Interleukin-1, Fc $\gamma$, vitamin-D receptor

It is largely accepted that periodontitis is heavily influenced by host genetic determinants. ${ }^{4}$ Gene polymorphisms expressing the basic aspects of the immune response, such as IL-1, Fc $\gamma$ and vit-D receptors, may be implicated. Studies on the genes themselves have proven shallow because of a lack of adjustment for important co-variables and the need for larger samples. Other risk assessments have neglected this crucial intrinsic aspect of the pathogenesis of periodontitis. $^{21,55}$

\section{PERIODONTITIS - RISK FACTOR FOR SYSTEMIC DISEASES?}

Where a patient's entire dentition (28 teeth) is affected by periodontitis, the subgingival surface area exposed to bacterial endotoxins has been likened to the entire inner surface of one's forearm. ${ }^{56}$ Such a potential focus of infection has justifiably raised concern over the possibility of systemic manifestations.

\section{Infective endocarditis (IE)}

Oral streptococci, particularly Streptococcus sanguis, are among the most common bacteria isolated from the blood of patients affected by Subacute IE following dental extractions and other procedures. ${ }^{57}$ This has led to the adoption of prophylactic measures to prevent bacteraemia. The evidence suggesting that periodontitis with mastication alone can produce sufficient bacteraemia to cause an endocarditis is equivocal. ${ }^{58}$ The consensus is that the majority of bacterial infections of prosthetic devices are of non-oral origin. ${ }^{59}$

\section{Coronary Heart Disease (CHD)}

Periodontitis and CHD may share a common aetiological pathway in the form of a hyperinflammatory monocyte phenotype that may account for an overexpression of inflammatory cytokines such as interleukin-1 $\beta$, tumour-necrosis factor- $\alpha$ and prostaglandin $E_{2}$ in both conditions. ${ }^{60}$ Streptococcus sanguis may also play a thrombogenetic role by way of causing accelerated platelet aggregation in response. ${ }^{61}$ Animal studies have been suggestive of such a relationship.

\section{Diabetes mellitus}

Largely accepted as a risk factor for periodontal disease, the idea is recent that diabetes may be complicated by periodontal infection. A mechanism explaining the physiological interaction between the two conditions involves an increase in glycated haemoglobin (accepted as the cause of many diabetic complications) in periodontal infection. ${ }^{62}$ Heightened immune reactivity, possibly spawned from a periodontal bacterial load, can lead to a predictably worsened diabetic state, such as overt nephropathy or end stage renal disease. ${ }^{63}$ Early reports have indicated that treatment of chronic periodontitis may reduce the need for supplemental insulin therapy, supporting the theory that periodontitis and diabetes interact in a cyclical pattern, rather than the former simply being an effect of the latter. ${ }^{64,65}$

\section{Pregnancy complications}

Speculation has led to the investigation of the effects of infection in pregnancy. It was suggested that inflammatory mediators normally involved in childbirth that are systemically raised due to periodontal infection (prostaglandin $\mathrm{E}_{2}$ and tumour necrosis factor- $\alpha$ ) may induce labour. ${ }^{66}$ Studies have correlated mothers of premature low birth weight infants as having more AL. ${ }^{67}$ 


\section{Respiratory diseases}

Respiratory pathogens are cultivated in the oral cavity, particularly following antibiotic therapy. ${ }^{68}$ Dental plaque in patients with poor oral hygiene may act as a reservoir for respiratory pathogens. ${ }^{69}$ The direct relationship between the two diseases has to be explored with respect to confounding factors. The plausibility of such a relationship is largely theoretical.

\section{Osteonecrosis}

Periodontal pathogens may create an anaerobic environment that facilitates the onset of osteochemonecrosis in individuals receiving bisphosphonate treatment (for osteoporosis or neoplastic bony metastasis), with some $84 \%$ of cases reporting comorbidity with periodontal disease. ${ }^{70}$ The role of periodontal infection on a previously sterile osteoradionecrosis has been described as contributory. ${ }^{71}$ With the hypocellularity and hypovascularity of the post-irradiation mandible, it seems likely that chronic infections in the periodontium and trauma during periodontal therapy may precipitate non-healing bony exposures. The studies highlight the necessity for pretreatment planning for teeth of poor prognosis. Further data are needed to quantify these risks. ${ }^{72}$

\section{DISCUSSION}

There are many factors thought to influence the incidence and progression of periodontal disease. The testing process is rigorous and comprehensive. Few factors have been unequivocally confirmed as true risk factors, though many more are known to exert some effect. It is in combination, building a profile of an individual, that one is able to evaluate a patient using a risk model.

Reviewing the literature establishes some factors as more likely risk predictors than others. Male patients with advanced age, lower SES and Asian, Pacific Island or Native American ethnicity are accepted as carrying a higher risk than a young, affluent, Caucasian female. A genetic component is accepted, though initial studies have proven inconclusive. Modifiable factors known to contribute include diseases, such as diabetes and obesity, behaviours such

\section{Table 1 Estimated risk of attachment loss, a product of risk factors}

\section{Range of Odds Ratios}

\begin{tabular}{|l|l|l|}
\hline & Low Estimate & High Estimate \\
\hline Age $65+$ & $3.40^{16}$ & $9.01^{17}$ \\
\hline Male & $1.36^{17}$ & $2.00^{19}$ \\
\hline Ethnicity* & $1.72^{20}$ & $2.40^{17}$ \\
\hline No college** & $2.60^{15}$ & $4.50^{22}$ \\
\hline Diabetic & $2.32^{30}$ & - \\
\hline Smoker & $1.45^{19}$ & $6.15^{15}$ \\
\hline Tannerella forsythensis & $2.40^{44}$ & $2.45^{20}$ \\
\hline Porphyromonas gingivalis & $1.59^{20}$ & $6.41^{14}$ \\
\hline BMI >30 & $1.76^{33}$ & $1.77^{34}$ \\
\hline Poor coping under stress & $1.71^{52}$ & $2.24^{51}$ \\
\hline $\begin{array}{l}* \text { Asian, Pacific Islander, Native American } \\
\text { *Refers to never having attended any third level education }\end{array}$ & & \\
\hline
\end{tabular}

as smoking, and cognitive factors such as poor coping behaviour. The disease is highly dependent on several microorganisms in both incidence and progression. With this in mind, the profile of a subject who would be most at risk of periodontitis (AL) is outlined (Table 1). The $O R$ of periodontitis for a person exhibiting all the above factors would naturally be compounded. Factors such as smoking, poor glycaemic control and poor oral hygiene account for a significant portion of this risk, and constitute the most modifiable and treatable portion of the risk profile. Smoking cessation, control of the diabetic state and the introduction of a proper hygiene regiment may minimise the effects of these considerable risks.

The suggestions of a causative role for periodontal infection in several systemic diseases are interesting and ever evolving. Many plausible pathways exist implicating periodontitis in the causation and/or exacerbation of infective endocarditis, coronary heart disease, diabetes mellitus, respiratory diseases, pregnancy complications or osteonecrosis, in some cases compellingly so. The lack of significant data expressing these risks in multivariate studies makes it difficult to concur with the conclusions of those investigations, but nonetheless raises interesting possibilities about the importance of good preventive dental care.
Additional investigation is advised to elucidate the relationships of these factors with periodontitis. The literature reviewed was almost exclusively cross sectional in nature. To further evaluate risk factors, studies need to be more prospective and longitudinal in form. The information gathered tended towards coincidental observations of disease and risk factor. Judgements regarding causation or contribution, without follow up examinations, would be misguided. An unfortunate limitation of having a large sample group is that recall and re-evaluation becomes problematic.

In the study of this disease, odds ratio helps to standardise risk assessment, allowing factors to be easily compared using a standardised numerical index. The benefits are irrefutable. Where the technique falls short is in the inconsistency of classification of 'cases' versus 'non-cases' of periodontitis. This binary system leaves little room for wider interpretation. Clinically, however, such a judgement is unavoidable. We thus support the view that criteria for a 'periodontitis case', as well as for 'disease progression' would enable clearer comparison of the examined risk indicators, with aspirations to further elucidate the extent of these influences. A mechanism would be to implement a 'scoring system' for periodontal risk factors. Below is our suggestion: 
Using odds ratio (Table 1), it is possible to construct a scoring system using the lower end estimated OR as a conservative figure. Collating the odds ratio for easy to examine demographic factors, the system becomes:

B BMI (high, >30)

score 2

E Ethnicity* score 1.5

D Diabetic (uncontrolled, type I/II)

score 2.5

S Stressed (poor coping under strain)

score 2

C College (none) $)^{* *}$

score 2.5

H Hygiene

(high plaque level)

score 2

A Age 65+

score 3.5

S Smoker

score 1.5

M Male

score 1.5

*Asian, Pacific Islander, Native American Cronin/Stassen Scale ${ }^{*}$ Refers to never having attended any third level education

Use of this mnemonic would make it easy for clinicians to summarise an individual's risk as a sum of their demography and clinical parameters, with a maximal risk score of 19 indicating an individual of maximal risk. This would allow clinicians to quickly and non-invasively identify those at risk, and if necessary recommend supportive or preventive therapies. ${ }^{73}$ Given that those who fit into more categories will be much more likely to experience the disease symptoms, there would be no harm in pursuing a policy of aggressive prevention for those fitting into a 'special risk' status. The benefits of early treatment of periodontal disease could not only reduce the need for invasive surgical or non-surgical periodontal treatment (in itself a worthwhile achievement), but could in doing so reduce the risk of various other systemic complication to which a periodontal disease sufferer would otherwise be predisposed. Given that the scale was produced as a product of literature review rather than clinical sampling, it would be recommended to validate the scale using a clinical trial. Cross-sectional study may reveal where on the 19 point risk scale the 'special risk' status may be suitably gauged.

1. Enwonwu C O, Edozien J C. Epidemiology of periodontal disease in western Nigeria in relation to socioeconomic status. Arch Oral Biol 1970; 15: 1231-1244.

2. Honkala E, Freeman R. Oral hygiene behaviour and periodontal status in European adolescents: an overview. Community Dent Oral Epidemiol 1988 16: 194-198.

3. Kingman A. Statistical management of periodontal data. Periodontol 2000 1993; 2: 83-97.

4. Loe $H_{1}$ Theilade $E_{1}$ Jensen $S B$. Experimental gingivitis in man. J Periodonto/ 1965: 36: 177-187.

5. Loe $\mathrm{H}$ et al. Natural history of periodontal disease in man. J Clin Periodontol 1986; 13: 431-440.

6. Kleinbaum D, Kupper L, Morgenstern H. Epidemiologic research: principles and quantitative methods. p 3. Belmont, California: Lifetime Learning Publications, 1982

7. Dark G (ed). Odds ratio from On-line Medical Dictionary. January2003.

8. Beck J D. Methods of assessing risk for periodontitis and developing multifactoral models. J Periodontol 1994; 65: 468-478.

9. Beck J D, Kohout F, Hunt R J. Identification of high caries risk adults: attitudes, social factors and diseases. Int Dent J 1988; 38: 231-238.

10. Tonetti M S, Claffey N. Advances in the progression of periodontitis and proposal of definitions of a periodontitis case and disease progression for use in risk factor research. J Clin Periodontol 2005; 32: $210-213$.

11. Lilienfeld A M. Foundations of epidemiology. pp 255-262. New York: Oxford University Press, 1976

12. Hill A B. Principles of medical statistics, 9 th ed. New York: Oxford University Press, 1971

13. Davies H T O, Crombie K, Tavakoli M. When can odds ratios mislead? BMJ 1998; 316: 989-991.

14. Pfeilschifter J et al. Mitogenic responsiveness of human bone cells in vitro to hormones and growth factors decreases with age. J Bone Miner Res 1993; 8: 707-717.

15. Beck J D et al. Prevalence and risk indicators for periodontal attachment loss in a population of older community-dwelling blacks and whites. J Periodontol 1990; 61: 521-528.

16. Locker $\mathrm{D}$, Leake J L. Risk indicators and risk markers for periodontal disease experience in older adults living independantly in Ontario, Canada. J Dent Res 1993b; 72: 9-17.

17. Grossi $S \mathrm{G}$ et al. Assessment of risk for periodontal disease. II. Risk indicators for alveolar bone loss. J Periodontol 1995; 66: 23-29.

18. Papapanou P N et al. Considerations on the contribution of ageing to loss of periodontal tissue support. J Clin Periodontol 1991; 18: 611-615.

19. Mumghamba E G S, Markkanen H A, Honkala E. Risk factors for periodontal diseases in Ilala, Tanzania. J Clin Periodontol 1995; 22: 347-354.

20. Horning G M, Hatch C L, Cohen M E. Risk indicators for periodontitis in a military treatment population. J Periodonto/ 1992;63: 297-302.

21. Borrell L N, Papapanou P N. Analytical epidemiology of periodontitis. J Clin Periodontol 2005; 32: 132-158

22. Susin C, Albandar J M. Aggressive periodontitis in an urban population in southern Brazil. J Periodontol 2005; 76: 468-475.

23. Mealey B et al. American Academy of Periodontology Position Paper - Diabetes and periodontal diseases. J Periodonto/ 2000; 71: 664-678.

24. Schmidt A M et al. Advanced glycation endproducts (AGEs) induce oxidant stress in the gingiva: a potential mechanism underlying accelerated periodontal disease associated with diabetes. J Periodontal Res 1996; 31: 508-515.

25. Taylor G W et al. Glycaemic control and alveolar bone loss progression in type 2 diabetes. Ann Periodontol 1998a; 3: 30-39.

26. Taylor $\mathrm{G}$ W et al. Non-insulin dependent diabetes mellitus and alveolar bone loss progression over 2 years. J Periodonto/ 1998b; 69: 76-83.

27. Rylander $\mathrm{H}$ et al. Prevalence of periodontal disease in young diabetics. J Clin Periodonto/ 1987; 14: 38-43.

28. Atkinson M A, Maclaren N K. What causes diabetes? Sci Am 1990; 263: 62-63, 66-71.

29. Thorstensson H, Kuylenstierna J, Hugoson A.
Medical status and complications in relation to periodontal disease experience in insulin dependent diabetics. J Clin Periodonto/ 1996: 23: 194-202.

30. Grossi S G et al. Assessment of risk for periodontal disease. I. Risk indicators for attachment loss. J Periodontol 1994; 65: 260-267.

31. Tervonen T, Karjalainen K. Periodontal disease related to diabetic status. A pilot study of the response to periodontal therapy in Type 1 diabetes. J Clin Periodontol 1997; 24: 505-510.

32. Seymour R A, Steele J G. Is there a link between periodontal disease and coronary heart disease? Br Dent J 1998; 184: 33-38.

33. Al-Zahrani M S, Bissada N F, Borawski E A. Obesity and periodontal disease in young, middle-aged, and older adults. J Periodonto/2003; 74: 610-615.

34. Linden G, Patterson C, Evans A et al. Obesity and periodontitis in 60-70-year-old men. J Clin Periodontol 2007; 34: 461-466.

35. Winkler J R, Murray P A. Periodontal disease. A potential intraoral expression of AIDS may be rapidly progressive periodontitis. J CalifDent Assoc 1987; 15: 20-24.

36. Chapple I L, Hamburger J. The significance of oral health in HIV disease. Sex Transm Infect 2000; 76: 236-243.

37. Scheutz $\mathrm{F}$ et al. Is there an association between periodontal condition and HIV infection? J Clin Periodontol 1997; 24: 580-587.

38. Yoshihara A et al. A longitudinal study of the relationship between periodontal disease and bone mineral density in community-dwelling older adults. J Clin Periodontol 2004; 31: 680-684.

39. Weyant R J et al. The association between osteopenia and periodontal attachment loss in older women. J Periodontol 1999; 70: 982-991.

40. Pindborg J J. Tobacco and gingivitis. 1. Statistical examination of the significance of tobacco in the development of ulceromembranous gingivitis and in the formation of calculus. J Dent Res 1947; 26: $261-264$

41. Baab D A, Öberg P A. The effect of cigarette smoking on gingival blood flow in humans. J Clin Periodonto/ 1987: 14: 418-424.

42. Feldman R S, Bravacos J S, Rose C L. Association between smoking different tobacco products and periodontal disease indexes. J Periodonto/ 1983; 54: 481-487.

43. Lindhe J, Karring T, Lang N P. Clinical periodonto/ogy and implant dentistry, 4th ed. Copenhagen: Blackell Munksgard, 2003.

44. Tezal M, Scannapieco F A, Wactawski-Wende J et al. Supragingival plaque may modify the effects of subgingival bacteria on attachment loss. J Periodontol 2006; 77: 808-813.

45. Papapanou P N et al. Sungingival microbiota in adult Chinese: prevalence and relation to periodontal disease progression. J Periodontol 1997; 68: 651-666.

46. Papapanou P N et al. Periodontal microbiota and clinical periodontal status in a rural sample in southern Thailand. Eur J Oral Sci 2002; 110: 345-352.

47. Loos B, Claffey N, Egelberg J. Clinical and microbiological effects of root debridement in periodontal furcation pockets. J Clin Periodontol 1988; 15: 453-463.

48. Geiger A M, Wasserman B H, Turgeon L R. Relationship of occlusion and periodontal disease. 8. Relationship of crowding and spacing to periodontal destruction and gingival inflammation. J Periodonto/ 1974; 45: 43-49.

49. Ainamo J. Malalignment of the teeth in relation to periodontal disease, abst. J Dent Res 1971; 50: 1220 .

50. Genco R J et al. Models to evaluate the role of stress in periodontal disease. Ann Periodontol 1998: 3: 288-302

51. Genco R J et al. Relationship of stress, distress and inadequate coping behaviors to periodontal disease. J Periodontol 1999; 70: 711-723.

52. Akhter R, Hannan M A, Okhubo R et al. Relationship between stress factor and periodontal disease in a rural area population in Japan. Eur J Med Res 2005; 10: 352-357.

53. Bernhardt 0 et al. The influence of dynamic 
occlusal interferences on probing depth and attachment level: results of the Study of Health in Pomerania (SHIP). J Periodontol 2006

77: 506-516.

54. Houston F et al. Mandibular dysfunction and periodontitis. A comparative study of patients with periodontal disease and occlusal parafunctions. Acta Odontol Scand 1987; 45: 239-246.

55. Loos B G, John R P, Laine M L. Identification of genetic risk factors for periodontitis and possible mechanisms of action. J Clin Periodontol 2005; 32: 159-179.

56. Waite D E, Bradley R E. Oral Infections. J Am Dent Assoc 1965; 71: 587-592.

57. LaCassin F, Hoen B, Leport $C$ et al. Procedures associated with infective endocarditis in adults. A case control study. Eur HeartJ 1995: 16: 1968-1974.

58. Murphy A M, Daly C G, Mitchell D H et al. Chewing fails to induce oral bacteraemia in patients with periodontal disease. J Clin Periodonto/ 2006; 33: $730-736$

59. Wahl M J. Clinical issues in the prevention of dental-induced endocarditis and prosthetic joint infection. Pract Periodontics Aesthet Dent 1995; 7: 29-36.

60. Shapira L, Soskolne W A, Sela M N et al. The secretion of $\mathrm{PGE}_{2}, \mathrm{IL}-1 \beta, \mathrm{IL}-6$ and TNF- $\alpha$ by monocytes from early onset periodontitis patients. J Periodontol 1994; 65: 139-146.

61. Herzberg M C, Meyer M W. Effects of oral flora on platelets: possible consequences in cardiovascular disease. J Periodontol 1996; 67: 1138-1142.

62. Taylor G W, Burt B A, Becker M P et al. Severe periodontitis and risk for poor glycaemic control in subjects with non-insulin-dependent diabetes mellitus. J Periodonto/ 1996; 67: 1085-1093.

63. Shultis W A, Weil E J, Looker H C et al. Effects of periodontitis on overt nephropathy and end-stage renal disease in type 2 diabetes. Diabetes Care 2007; 30: 306-311.

64. Williams R C, Mahan C J. Periodontal disease and diabetes in young adults. JAMA 1960; 172: 776-778.

65. Miller LS, Manwell M A, Newbold D et al. The relationship between reduction in periodonta inflammation and diabetes control: a report of 9 cases. J Periodontol 1992; 63: 843-849.

66. Scannapieco F A et al. Position paper. Periodontal disease as a potential risk factor for systemic diseases. J Periodonto/ 1998; 69: 841-850.

67. Offenbacher S, Katz V L, Fertik G S et al. Periodontal infection as a risk factor for preterm low birth weight. J Periodontol 1996; 67: 1103-1113.

68. Helovuo H, Hakkarainen K, Paunio K. Changes in the prevalence of subgingival enteric rods, staphylococci and yeasts after treatment with penicillin and erythromycin. Oral Microbiol Immunol 1993; 8: 75-79.

69. Komiyama K, Tynan J J, Habbick B F et al. Pseudomonas aeruginosa in the oral cavity and sputum of patients with cystic fibrosis. Oral Surg Oral Med Oral Pathol 1985; 59: 590-594.

70. Marx R E, Sawatari Y, Fortin M et al. Bisphosphonate-induced exposed bone (osteonecrosis/osteopetrosis) of the jaws: risk factors, recognition prevention, and treatment. J Oral Maxillofac Surg 2005; 63: 1567-1575.

71. Galler C, Epstein J B, Guze K A et al. The development of osteoradionecrosis from sites of periodontal disease activity: report of 3 cases. J Periodontol 1992; 63: 310-316.

72. Flint $S R$, Sharkey S, Galvin S. Warning: bisphosphonates and osteochemonecrosis of the jaws. $J$ Ir Dent Assoc 2006; 52: 79-83.

73. al-Qurainy I A, Titterington D M, Dutton G N et al. Midfacial fractures and the eye: the development of a system for detecting patients at risk of eye injury. Br J Oral Maxillofac Surg 1991; 29: 363-367. 